\title{
A selected review of abstracts from the 20th Annual Meeting of the Society for Neuro-Oncology (SNO)
}

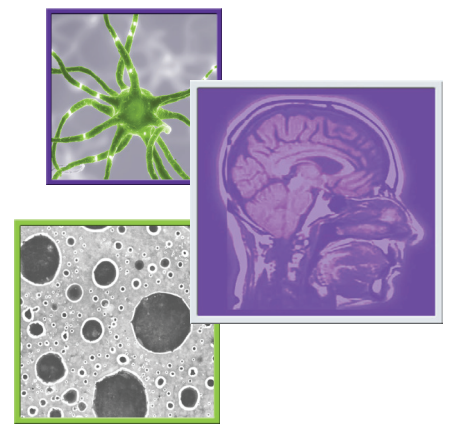

\author{
Marc C Chamberlain*
}

\section{0th Annual Meeting of the Society for Neuro-Oncology, San Antonio, TX, USA, 18-22 November 2015}

The Society for Neuro-Oncology is the largest neuro-oncology meeting in the USA that meets annually and provides a multiday venue that showcases new brain cancer clinical trial results and basic research primarily pertaining to gliomas. The Society for Neuro-Oncology 2015 meeting comprising one education day, 2 days of premeetings and 3 days of presentation, over 200 oral presentations and 900 abstracts provides an overview of contemporary neurooncology that includes metastatic disease of the central nervous system as well as primary brain tumors. This review attempts to highlight select abstracts presented at this year's meeting in a short summary that provides a synopsis of a large and multifaceted meeting.

\section{Metastatic disease to the CNS}

\section{- Parenchymal brain metastases}

A study by the Alliance consortium evaluated in a randomized trial stereotactic radiosurgery (SRS) with or without whole brain radiotherapy (WBRT) in 213 patients with 1-3 brain metastases (BM) (68\% lung cancer related) [1]. The primary end point was cognitive progression at 3 months using six cognitive test instruments. Cognitive progression at 3 months (as reflected by immediate recall, delayed recall and verbal fluency) was more frequent following SRS + WBRT compared with SRS only (92 vs 64\%). Intracranial tumor control at 3 and 6 months were better in the SRS + WBRT cohort (75.3 and $64.7 \%$ with SRS alone vs 93.7 and $88.4 \%$ with SRS + WBRT), however, overall survival (OS) was similar in both groups. The authors conclude that in patients with 1-3 BM, SRS only is the preferred therapy with less cognitive impact and similar survival. Not clear, however, from this study was whether the deterioration in cognitive function seen with SRS + WBRT was maintained at 6 months or were there data regarding progressive neurological disease as a cause of death in the two treatment arms. Essentially all studies in BM have failed to show a difference in OS and consequently the significance of this as an end point in $\mathrm{BM}$ trials is unclear. Lastly, there were no data regarding the need for salvage therapy in patients treated with SRS only.

A study of 51 patients with ALK-rearranged non-small-cell lung cancer and BM all of whom had progressed on crizotinib, an ALK inhibitor, were treated with the recently approved ALK inhibitor alectinib [2]. Patients with ALK + NSCL and BM were a subset of all patients enrolled in one of two prospective Phase II trials of alectinib. The intracranial response was $61 \%$ and median

*Department of Neurology/Division of Neuro-Oncology, University of Washington, Fred Hutchinson Cancer Research Center, Seattle Cancer Care Alliance, 825 Eastlake Avenue E, POB 19023, MS G4940, Seattle, WA 98109-1023, USA; Tel.: +1 2062888280 ; Fax: +1 206288 2000; chambemc@u.washington.edu

\section{KEYWORDS}

- primary and secondary (metastatic) brain tumors - SNO 2015 abstracts 
duration of response was 9 months (regardless of response criteria utilized, RECIST vs RANO) suggesting sequential treatment with ALK inhibitors can effectively control BM in ALK-rearranged non-small-cell lung cancer BM.

\section{- Leptomeningeal metastases}

Le Rhun et al. presented early data $(\mathrm{n}=20)$ from the French Deposein trial in patients with breast cancer and leptomeningeal metastases (LM) [3]. The study compares systemic therapy only to systemic therapy + intra-cerebrospinal fluid (CSF) liposomal cytarabine. LMD was defined by positive CSF, MRI compatible with LM or both. Similar toxicity was seen in both arms of the study. The trial continues to accrue and when complete will comprise the largest randomized prospective study that evaluates the role of intra-CSF chemotherapy in LMD.

\section{Primary brain tumors}

\section{- Meningiomas}

Grimm et al. presented the results of a Phase II trial in surgery and radiation refractory meningioma exploring the utility of bevacizumab [4]. Best responses were stable disease (WHO grade I [ $\mathrm{n}=15]$ ]: $100 \%$; grade II [ $\mathrm{n}=22$ ]: $86 \%$; grade III $[\mathrm{n}=13]: 82 \%$ ); partial response (grade I: $0 \%$; grade II: $5 \%$; grade 3: $0 \%$ ), progressive disease (grade I: $0 \%$; grade II: $5 \%$; grade III: $18 \%)$. PFS-6, mPFS and mOS were $87 \%$, 22.5 months, 35.6 months for grade I; $77 \%$, 15.3 months, not reached for grade II, and $46 \%, 3.7$ months, 12.4 months for grade III. The results are promising compared with a contemporary meta-analysis, in which PFS6 was $29 \%$ and $27 \%$ for grade I and grades II/III respectively but require confirmation.

Seystahl et al. reported on somatostatin receptor targeted radionuclide therapy for progressive meningioma (all grades) using the radio-isotopes 90Y-DOTATOC or $177 \mathrm{Lu} 0$ DOTATAE in 20 patients [5]. Somatostatin receptor-targeted radionuclide treatment appeared safe and is effective in a subset of patients with WHO grade I and II meningioma requiring systemic therapy, but has limited efficacy in patients with WHO grade III meningioma.

\section{- Primary CNS lymphomas}

Chamberlain presented a small retrospective case series of patients $(n=13)$ with recurrent PCNSL treated with high-dose araC (HD-ara-C) and demonstrated minimal radiographic response and progression-free survival [6]. These findings replicated those of the German PCNSL trial in which newly diagnosed patients with PCNSL refractory to HD-MTX were treated with $\mathrm{HD}$-araC in which minimal response was observed. These studies question the utility of HD-araC in PCNSL.

\section{- Gliomas}

Low-grade glioma

Up-front trials

Buckner et al. presented molecular pathology from the randomized Phase III Radiation Treatment Oncology Group (RTOG 9802) trial in high-risk low-grade glioma (LGG) (age $>40$ years or incomplete resection) comparing up-front RT (radiation therapy) only to RT + PCV (procarbazine, CCNU, vincristine) chemotherapy [7]. Two hundred and fifty-one patients (107 [43\%] oligodendroglioma, 79 [31\%) oligoastrocytoma, 65 astrocytoma [26\%]) were accrued to trial and 113 were analyzable for IDH1 mutation. Seventy-one of 113 highrisk LGG showed the IDH1 mutation in whom median PFS and OS was superior to IDH1 wildtype tumors (7.6 vs 1.5 years; 5.1 vs 13.1 years) irrespective of treatment. Furthermore RT + PCV resulted in superior survival outcomes when compared with RT only in IDH1 mutated LGG. Similar to RTOG 9813 discussed below, the presence of IDH1 wild-type tumor predicts for impoverished survival regardless of treatment.

\section{Anaplastic astrocytoma}

Up-front trials

Chang et al. presented the NRG Oncology/ RTOG 9813 trial that compared TMZ (temozolomide) to a nitrosourea (BCNU or CCNU) following maximal safe resection and RT in newly diagnosed anaplastic astrocytoma (AA) [8]. Although the study was closed prematurely due to poor accrual, 206 patients were entered and randomized and represents the only trial to prospectively compare alkylating chemotherapies in newly diagnosed AA. Toxicity differed between the study arms wherein nitrosoureas were both more toxic (grade $>2$ seen in 75 vs $47 \%$ ) and led to higher discontinuance of therapy due to toxicity ( $27 \mathrm{vs} 0 \%$ ). Notwithstanding toxicity differences, both TMZ and BCNU/CCNU were comparable 
with respect to median PFS and OS. The most notable difference was seen in median OS in patients whose tumor was IDH1 mutant (52\%) compared with IDH1 wild-type tumors (49\%; 7.9 vs 2.8 years). Although not presented at SNO, early analysis of the CATNON trial suggests 12 months of post-RT TMZ is superior to no post-RT chemotherapy following surgery and RT (with or without concurrent TMZ) in noncodeleted anaplastic gliomas. Further analysis of CATNON will likely inform as to best therapy for IDH1 mutant (RT+TMZ as is now recommended) versus wild-type (possibly RT only?) noncodeleted anaplastic gliomas (AG).

Jaeckle $e t a l$. presented preliminary data from the CODEL trial in newly diagnosed $1 \mathrm{p} 19 \mathrm{q}$ codeleted AG. The trial was originally designed as a randomized three-arm study (TMZ only, RT only and RT + TMZ) where however only 50 patients were to be entered on the TMZ only arm [9]. In early results, the study demonstrated inferior outcome in the TMZ only arm with respect to PFS, neurocognitive progression and death due to progressive disease. As a consequence, CODEL was redesigned and based on mature results from RTOG 9402 and EORTC 25691 as well as RTOG 9802, is comparing RT + PCV to RT + TMZ.

\section{Glioblastoma}

Up-front trials

A pooled retrospective analysis of four randomized clinical trials (EORTC/NCIC 26981; EORTC26071-CENTRIC; EMD-CORE; and RTOG 0525-Intergroup) did not show a survival benefit if post-RT TMZ was extended beyond six cycles. This finding was true regardless of the tumor MGMT promoter methylation status [10]. This analysis would suggest little to no benefit in administering TMZ beyond 6-month post-RT in newly diagnosed glioblastoma (GBM).

The compound ICT-107, a vaccine of patientderived dendritic cells incubated with peptides derived from six tumor-associated antigens (MAGE-1, HER2, AIM-2, TRP-2, gp100 and IL-13R $\alpha 2$ ) showed in a small Phase II trial in patients with newly diagnosed glioblastoma a significantly improved PFS in the experimental arm [11]. In the subgroup of HLA-A2-positive patients, immunologic response was associated with a median OS of 23.1 months for responders and 13.7 for nonresponders. Based on these encouraging results, a multicenter randomized, double-blind Phase III trial in HLA-A2-positive patients with newly diagnosed glioblastoma has been initiated.

Zhu et al. presented the quality of life assessment using the EORTC QLQ C-30/BN20 instrument in the EF-14 trial comparing SOC with or without tumor-treating fields and reported there was no adverse effect of tumortreating fields when compared with TMZ only [12].

\section{Salvage trials}

Gan et al. reported the Phase I results of ABT414, a monoclonal antibody-drug conjugate targeting EGFR expressing GBM [13]. The recommended Phase II dose was established $(1.25 \mathrm{mg} / \mathrm{kg})$ either as monotherapy or in combination with TMZ. Unique drug-related toxicity was ocular (keratitis), hepatic (GGTP increase) and thrombocytopenia. Responses were noted only in tumors amplified for EGFR. A randomized Phase III trial in newly diagnosed GBM amplified for EGFR is planned based on these studies.

Brenner et al. presented a Phase II trial of VB-111, an antiangiogenic agent consisting of a nonreplicating adenovirus vector with a modified preproendothelin promoter that leads to apoptosis of tumor vasculature by expressing a fas-chimera transgene in angiogenic endothelial cells [14]. VB-111 was given in conjunction with bevacizumab for recurrent GBM and results suggest an improvement over bevacizumab monotherapy (16 vs 8 months median OS). This novel intravenous agent is proceeding to a randomized Phase III trial in newly diagnosed GBM.

Of the several presentations of TOCA 511, a retroviral replicating vector encoding yeast cytosine deaminase, administered in combination with extended release 5-flurocytosine, a novel intravenous Phase I trial was notable for obviating the usual administration of TOCA 511 by intratumoral or convection enhanced delivery [15]. Patients scheduled to undergo a planned reresection for recurrent GBM were first given TOCA 511 intravenously and a planned surgical analysis (presence of virus and the transgene cytosine deaminase) was performed as a proof of effective delivery. The study demonstrated viral DNA in tumor in a dose-dependent manner as well as presence of the transgene. Further studies are planned using the intravenous delivery system.

Based upon results of the Dutch BELOB trial, EORTC-2610l was initially planned as a 
Phase II study with four arms (bevacizumab followed by CCNU, CCNU followed by either bevacizumab or best investigator's choice or the combination of both followed by best-investigator's choice after progression on the first regimen). In the adapted Phase III trial design, the combination of bevacizumab and CCNU was compared with CCNU as a single agent in patients with GBM and first recurrence [16]. The primary end point, OS, was not significantly different between CCNU monotherapy (median OS 8.6 months, $\mathrm{n}=149$ patients) and the combination with bevacizumab (9.1 months, $\mathrm{n}=288$ patients, hazard ratio [HR]: 0.95). PFS was longer in the combination arm (4.2 months, HR: 0.49) compared with CCNU alone (1.5 months). Crossover to bevacizumab in the monotherapy arm occurred in $35.5 \%$ while $19 \%$ of the patients in the combination arm continued bevacizumab after tumor progression. EORTC-26101 raises the question of whether bevacizumab adds benefit relative to CCNU in patients with recurrent GBM and the trial results potentially jeopardize the current US FDA approved indication for bevaciaumab in recurrent GBM.

Lee et al. presented results of the NRG/ RTOG 1122 randomized Phase II trial of bevacizumab with or without AMG 386, an angiopoietin binding fusion protein in recurrent GBM [17]. Like the majority of add-on bevacizumab trials, the experimental arm was not superior to single agent bevacizumab. Robbins et al. discussed the NRG/RTOG 0929 Phase I/ II study of ABT-888 (veliparib, a PARP inhibitor) in combination with $2 \mathrm{TMZ}$ treatment regimens (5/28 and 21/28) in recurrent TMZ resistant glioblastoma [18]. Both bevacizumab naive and experienced cohorts were evaluated and in both cohorts response rate was low and PFS-6, the primary end point failed to meet prespecified criteria. The study suggests inhibition of base excision repair with a PARP inhibitor does not restore TMZ sensitivity in recurrent GBM.

The Re-ACT trial randomized patients with recurrent GBM to bevacizumab plus placebo vaccine versus bevacizumab combined with the EGFRviii peptide vaccine, rindopepimut. Patients in the rindopepimut arm had higher overall response rates (30 vs $18 \%$ ), PFS-6 (28 vs $16 \%$ ) and prolonged median OS (11.3 vs 9.3 months, HR $=0.57$ ). Nonetheless, the trial failed to meet the prespecified study end point of PFS-6 greater than 40\% [19]. More compelling regarding the utility of rindopepimut in GBM will be final results of the now completed ACT 4 trial comparing standard of care with or with rindopepimut in newly diagnosed GBM.

A Phase III trial evaluating nivolumab, an antibody targeting PD-1, versus bevacizumab in patients with recurrent GBM completed accrual (Checkmate-143) and within a small safety cohort $(n=20)$ of patients enrolled, the OS rate at 6 months was $70 \%$ for patients receiving nivolumab alone [20]. Monotherapy of nivolumab was well tolerated while the combination with ipilimumab (the third arm of the study) was discontinued at Phase I because of limiting toxicity including colitis, cholecystitis, diabetic ketoacidosis and confusion. Nivolumab is to be evaluated in patients with newly diagnosed GBM in both unmethylated (Checkmate-498; SOC vs RT + nivolumab) and methylated tumors (Checkmate-548; SOC with or without nivolumuab).

In a preliminary report O'Rourke $e t$ al. demonstrated the safety and feasibility of administering CAR (chimeric antigen receptor) $T$ cells redirected to EGFRviii in patients with EGFRviii + recurrent GBM [21].

\section{Summary}

SNO 2015 had as highlights several trials that may be practice changing. These include RTOG 9813 wherein no difference in OS was seen in comparing post-RT TMZ to either BCNU or $\mathrm{CCNU}$ in newly diagnosed anaplastic astrocytoma. In recurrent GBM, the EORTC 26101 trial comparing bevacizumab with CCNU versus CCNU monotherapy suggests no difference in survival and may potentially challenge the current indication of bevaciaumab for this disease. Lastly, the Alliance trial comparing SRS to WBRT in patients with 1-3 BM suggests no difference in OS and less cognitive decrement in patients treated with SRS.

Financial \& competing interests disclosure

The author has no relevant affiliations or financial involvement with any organization or entity with a financial interest in or financial conflict with the subject matter or materials discussed in the manuscript. This includes employment, consultancies, honoraria, stock ownership or options, expert testimony, grants or patents received or pending, or royalties.

No writing assistance was utilized in the production of this manuscript. 


\section{References}

1 Brown P, Asher A, Ballman K et al. NCCTG N0574 (ALLIANCE): a phase III randomized trial of whole brain radiation (WBRT) in addition to radiosurgery (SRS) in patients with 1 to 3 brain metastases. Neuro-Oncol. 17(Suppl. 5), Abstract BMET-05 (2015).

2 Ou SA, Shaw A, Gandhi L et al. Assessing central nervous system (CNS) response to alectinib in two Phase II studies of pre-treated ALK+ non-small cell lung cancer (NSCLC): RECIST vs. RANO criteria. Neuro-Oncol. 17(Suppl. 5), Abstract BME-18 (2015).

3 Le Rhun E, Zairi F, Boulanger T et al. Results of the interim safety analysis of the DEPOSEIN trail in leptomeningeal metastases from breast cancer. Neuro-Oncol. 17(Suppl. 5), Abstract BMET-25 (2015).

4 Grimm S, Kumthekar P, Chamberlain M et al. Phase II trial of bevacizumab in patients with surgery and radiation refractory progressive meningioma. Neuro-Oncol. 17(Suppl. 5), Abstract MNGO-04 (2015).

5 Seystahl K, Stoecklein V, Schuller U et al. Somatostatin receptor targeted radionuclide therapy for progressive meningioma. Neuro-Oncol. 17(Suppl. 5), Abstract MNGO-20 (2015).

6 Chamberlain M. High-dose cytarabine salvage therapy for recurrent primary CNS lymphoma. Neuro-Oncol. 17(Suppl. 5), Abstract ATCT-10 (2015).

7 Buckner J, Shaw E, Pugh S et al. IDH1 R132H mutations in NRG/RTOG 9812: Phase III study of radiation therapy (RT) only versus RT plus procarbazine, CCNU, and vincristine $(\mathrm{PCV})$ in patients with low grade glioma. Neuro-Oncol. 17(Suppl. 5), Abstract ATCT-09 (2015).

8 Chang S, Zhang P, Cairncross G et al. Results of NRG/RTOG 9813- A Phase III randomized study of radiation therapy (RT) and temozolomide (TMZ) versus RT and nitrosoureas (NU) therapy for anaplastic astrocytoma (AA). Neuro-Oncol. 17(Suppl. 5), Abstract ATCT-12 (2015).

9 Jaeckle K, Vogelbaum M, Ballman K et al. CODEL (Alliance-N0577;

EORTC-26081/2208; NRG-1071; NCIC-CEC-2): Phase III randomized study of RT vs. RT + TMZ vs. TMZ for newly diagnosed 1p/19q codeleted anaplastic glioma. Analysis of patients treated on the original protocol design. Neuro-Oncol. 17(Suppl. 5), Abstract ATCT-16 (2015).

Blumenthal DT, Stupp R, Zhang P et al. The impact of extended adjuvant. temozolomide in newly-diagnosed glioblastoma: a secondary analysis of EORTC and. NRG Oncology/ RTOG. Neuro Oncol. 17(Suppl. 5), Abstract ATCT-08 (2015).

11 Wen P, Reardon D, Phuphanich S et al. Association of survival and progression free. survival with immune response in HLA-A2+ newly-diagnosed GBM patients in. randomized double-blind placebo-controlled Phase 2 trial of dendritic cell (DC). immunotherapy with ICT-107. Neuro Oncol. 17(Suppl. 5), Abstract IMCT-20 (2015).

12 Zhu JJ, Pannullo S, Mehdorn M et al. Quality of life, cognitive function and functional status in the EF-14 trial: a prospective multicenter trial of TTfields with temozolomide compared to temozolomide alone in patients with newly diagnosed GBM. Neuro-Oncol. 17(Suppl. 5), Abstract ATCT-35 (2015).

13 Gan HK, Kumthekar P, Lassman AB et al. ABT-414 monotherapy or combination therapy with temozolomide (TMZ) rechallenge in recurrent glioblastoma (GBM) and amplified epidermal growth factor receptor (EGFR): a Phase I study. NeuroOncol. 17(Suppl. 5), Abstract ATNT-01 (2015).

14 Brenner A, Cohen Y, Vredenburgh J et al. Phase 2 study of VB-111, an anti-cancer therapy as monotherapy followed by combination of VB-111 with bevacizumab in recurrent glioblastoma. Neuro-Oncol. 17(Suppl. 5), Abstract BMET-26 (2015).

15 Cloughesy T, Vogelbaum M, Ostertag D et al. Intravenous TOCA 511 delivery leads to viral DNA in resected HGG. Neuro-Oncol. 17(Suppl. 5), Abstract DDEL-07 (2015).

16 Wick W, Brandes A, Gorli T et al. Phase III trial exploring the combination of. bevacizumab and lomustine in patients with first recurrence of a glioblastoma: The. EORTC 26101 trial. Neuro Oncol. 17(Suppl. 5), Abstract LB-05 (2015).

17 Lee EQ, Zhang P, Wen PY et al. NRG/ RTOG 1122: Phase III double-blinded, placebo controlled study of bevacizumab with or without AMG 386 in patients with recurrent glioblastoma or gliosarcoma. Neuro-Oncol. 17(Suppl. 5), Abstract ATCT-22 (2015).

18 Robbins HI, Zhang P, Gilbert M et al. NRG/ RTOG 0929: a randomized PhaseI/II study of ABT-888 in combination with temozolomide in recurrent temozolomide resistant glioblastoma. Neuro-Oncol. 17(Suppl. 5), Abstract ATCT-27 (2015).

19 Reardon DA, Desjardins A, Schuster J et al. ReACT: long-term survival from a. randomized phase II study of rindopepimut (CDX-110) plus bevacizumab in relapsed. glioblastoma. Neuro Oncol. 17(Suppl. 5), Abstract IMCT-08 (2015).

20 Sampson J, Omuro A, Vlahovic G et al. Safety and activity of nivolumab monotherapy and nivolumab in with combination ipilimumab in recurrent glioblastoma (GBM): updated results from CHECKMATE-143. Neuro Oncol. 17(Suppl. 5), Abstract IMCT-03 (2015).

21 O'Rourke D, Desai A, Morrissette, et al. Pilot study of $T$ cells redirected to EGFRviii with a chimeric antigen receptor in patients with EGFRviii + glioblastoma. Neuro-Oncol. 17(Suppl. 5), Abstract IMCT-15 (2015). 